focus on German Studies.

https://journals.uc.edu/index.php/fogs

Issue 28 (2021)

\title{
Autopsie einer Schuld. Zu den Erzählweisen der Fallgeschichte in \\ Friedrich Schillers Der Verbrecher aus verlorener Ehre
}

\section{Oliver Sommer \\ Goethe-Universität Frankfurt am Main}

Abstract

Friedrich Schillers Der Verbrecher aus verlorener Ehre thematisiert die Erä̈hlweisen von Fallgeschichten. Dieser Beitrag arbeitet heraus, wie der Eræähler der Vorrede die kulturelle Praxis des Schreibens von historiographischen Texten problematisiert. Dazu vergleicht er sie mit (seinen eigenen) poetischen Erä̈hlweisen und wertet sie im Rückgriff auf seine moralisch-pädagogischen Bestrebungen ab. Endgültige oder eindeutige Erklärungen für bestimmte Fragestellungen (in diesem Fall solche der (Re-)Konstruktion einer historischen Persönlichkeit) qu entwickeln, ist ein Vorgang, den diese Poetik kritisch beleuchtet. Bei genauerer Betrachtung der von der Forschung bisher wenig untersuchten Zusammenhänge zwischen historiographischem und poetischem Eræählen auf der einen und den moralisch-pädagogischen Bemerkungen des Erzählers auf der anderen Seite hebt der Text dagegen mehrere, mehrdeutige und unabgeschlossene, wenngleich sich widersprechende Erzählungen über ein und denselben Sachverbalt positiv hervor.

Keywords:

Schiller - Der Verbrecher aus verlorener Ehre - Paradoxen - Historiographie

ORCID ID: https://orcid.org/0000-0003-0253-7306

How to cite: Sommer, Oliver. “Autopsie einer Schuld. Zu den Erzählweisen der Fallgeschichte in Friedrich Schillers Der Verbrecher aus verlorener Ehre“. focus on German Studies 28, no. 28, 2021, pp. 90-115. DOI: 10.34314/FOGS2021.00006 


\section{Autopsie einer Schuld. Zu den Erzählweisen der Fallgeschichte in}

\section{Friedrich Schillers Der Verbrecher aus verlorener Ehre}

\section{Oliver Sommer}

\section{Fallgeschichten und ihre paradoxen Verhältnisse}

Dieser Beitrag will zeigen, mittels welcher Verfahren in Schillers Erzählung Der Verbrecher aus verlorener Ehre solche Texte kritisch betrachtet werden, die eine Fallgeschichte präsentieren. Um diese Verfahren detailliert beschreiben zu können, verbinde ich sie mit einigen Überlegungen zum Begriff der ,Fallgeschichte‘ und beziehe sie daraufhin auf die Erzähl- und Schreibweisen im Verbrecher aus verlorener Ehre. Mein Beitrag ist in zwei Teile gegliedert: Zuerst gilt es, auf die Überlegungen des Erzählers zur Historiographie in der Vorrede der Erzählung einzugehen. Dabei wird sich zeigen, dass der Erzähler historiographische Erzählweisen thematisiert, um sie mit poetischen zu vergleichen. In einem zweiten Schritt, so meine erste These, beansprucht er bei zeitgleich erfolgender Kritik an der Geschichtsschreibung historiographische Verfahren für seine poetische Erzählweise, die insbesondere die erzählerische Gestaltung der Fallgeschichte über Christian Wolf prägen. Danach nehme ich die in der Vorrede ausgiebig genutzten moralisch-pädagogischen Bemerkungen genauer in den Blick. In der damit versuchten Lesart lenkt der Erzähler die Lektüre seiner Rezipient*innen, so meine zweite These, um ihnen klar zu machen, dass eine mehrdeutige Betrachtung von Verbrechen und Verbrecher*innen notwendig ist: In der Perspektive von Wolfs Fallgeschichte erkennen sie die menschlichen Züge einer tragischen Figur, die jedem*r noch so grausamen Verbrecher*in stets eigen sind. Zum Schluss gebe ich einen Ausblick, der kurz umreißt, welche Untersuchungen auf meinen Beitrag folgen können. 
focus on German Studies. https://journals.uc.edu/index.php/fogs (ISSN 1076-5697) focus on German Studies

Die Ambiguität von Fallgeschichten erläutert Susanne Lüdemann: Eine Geschichte ist nur dann ein Fall, wenn man sie mit anderen Geschichten vergleichen kann. Gemeinsam bilden die Geschichten einen Fall und teilen Merkmale, die ihn als spezifischen Fall begründen. Zugleich ist jede Fallgeschichte aber auch eine Ausnahme von der Regel und unterscheidet sich zumindest hinsichtlich eines Sachverhalts von denjenigen Merkmalen, die allen Geschichten eines Falls zukommen (Lüdemann 208).

Ich stimme Lüdemann zu, wenn sie das ,paradoxe Verhältnis zwischen Abweichung und Norm, Ausnahme und Regel oder Individuellem und Allgemeinem“ (209) zur Grundformel aller Fallgeschichten erklärt, doch ist das noch nicht alles. Denn gerade aufgrund dieses paradoxen Verhältnisses erzähle jede Fallgeschichte Nicolas Pethes zufolge etwas Neues mit „einem spezifischen Sensationswert“ (32), das sie besonders auszeichne und erst zum Fall werden lasse. Laut Arne Höcker vermitteln Fallgeschichten „ein erst noch zu etablierendes und somit prekäres Wissen“ (Der Fall der Literatur 68). Wenn Fallgeschichten ihren Rezipient*innen allerdings tatsächlich erkenntnis- oder wissensstiftende Erzählungen bereitstellen, müssen ihre Narrative - wie es Hayden White in seiner Kritik an historiographischen Erzählweisen formuliert hat in Zusammenhang gebracht werden. Die so entstandene Erzählung muss eine leicht zugängliche Geschichte einerseits, eine plausible Erklärung des vergangenen Geschehens andererseits sein (White 86). Steffen Martus argumentiert deshalb, dass Fallgeschichten, indem sie den individuellen Fall einer Figur erzählen, bei den Rezipient*innen auf Verständnis stoßen (252). Auch Pethes betont die gegen Ende des 18. Jahrhunderts in Fallgeschichten kommunizierte „Aufwertung des Status des Individuums“ (23). Das ist einleuchtend, weil poetische, historiographische, journalistische, juristische, psychologische oder andere Erzählweisen anhand von personalisierten Geschichten auch immer in sich geschlossene und daher leicht nachzuvollziehende Erzählungen hervorbringen können. Einen Schritt weiter geht Johannes Lehmann, der behauptet, dass eine solche verständliche Fallgeschichte nur dann gelinge, wenn sie nahbar sei, also wenn sie von einer glaubhaften Lebensgeschichte der Person oder Figur gerahmt sei, auf der der individuelle Fall beruhe (Erfinden, was der Fall 
focus on German Studies.

https://journals.uc.edu/index.php/fogs

focus

(ISSN 1076-5697)

on German Studies

ist 364). Höcker führt die für Fallgeschichten grundlegende Ausrichtung auf ein Allgemeines hauptsächlich auf diese Rahmung zurück (Der Fall der Literatur 69).

In Anlehnung an Lehmann ist ein erzähltheoretischer Zugang zu dieser Erzählung besonders interessant, weil für jede Fallgeschichte stets von Neuem zu prüfen ist, wie sich ihre, innere narrative Rahmung“(Erfinden, was der Fall ist 365) ausgestaltet. Meine zentrale Fragestellung lautet: Mit welchen Argumenten kritisiert der Erzähler der Vorrede in Der Verbrecher aus verlorener Ehre, dass bestimmte Erzählweisen einen Fall jeweils eindeutig erschließen? Schillers aus heutiger literaturtheoretischer Sicht als metanarrativ erscheinende Erzählung stellt einen geeigneten Schauplatz für die nachfolgenden Analysen bereit. Die darin erzählten Welten der Rahmen- und Binnenerzählung[en] behandeln einen Fall, der auf der inhaltlichen Ebene auf zur Entstehungszeit des Textes neue Kenntnisse zurückgreift, die sich aus der seinerzeit neuen Forschungsrichtung der ,Seelenkunde“ ergeben. Um Joachim Pfeiffer zu folgen, wird der Konflikt zwischen gesetzlicher Norm und der „Eigentümlichkeit der psychischen Struktur des Menschen“ (101) thematisiert. Darüber hinaus ist die Erzählung für die Analyse mittels erzähltheoretischer Ansätze geeignet, weil sich ihre narrative Struktur - wie Sophia Avgerinou hervorhebt - als mehrdeutig präsentiert (15).

Erforscht wurde der Text in vielerlei Hinsicht: Pfeiffers Gegenüberstellung von strafrechtlichem und anthropologischem Diskurs ist Thema zahlreicher Forschungsbeiträge. Viel Zustimmung bekam Eberhard Ostermanns anerkennungstheoretische Deutung der Erzählung, in der er für die Figur Christian Wolf feststellt, dass sie in gezielten Akten der Missachtung des herrschenden Normensystems ihre Selbstachtung zurückerlange (218). Weitestgehend ausgereizt ist auch das Thema der Subjektkonstitution dieser Figur, wie es beispielsweise Sara Bangert und Klaus Müller-Richter verfolgt haben.

All dies heißt allerdings nicht, dass weitere Analysen dem nichts mehr hinzuzufügen hätten. Da Schiller in Der Verbrecher aus verlorener Ehre eine Fallgeschichte im Medium des poetischen Textes und durch 
focus on German Studies. https://journals.uc.edu/index.php/fogs (ISSN 1076-5697) focus on German Studies

Rückgriff auf unterschiedliche Schreibweisen darstellt, soll hier noch einmal geprüft werden, wie er die ,Wahrheit ${ }^{\star}$ dieser „wahren Geschichte“ - so bereits der peritextuelle Hinweis im Untertitel - vermittelt. Der von Lüdemann herausgearbeiteten Funktion poetischer Texte, „gesellschaftliche] Normierungs- und Normalisierungsprozesse“(212) zu reflektieren, ist Höckers Forderung an die Seite zu stellen, den Blick stärker auch auf die ästhetischen bzw. narrativen Verfahren zu richten, mit denen die Erzähler in Der Verbrecher aus verlorener Ehre nicht nur eine beliebig erfundene, sondern eben auch eine historiographisch belegte Fallgeschichte präsentieren (Der Fall der Literatur 70). Gerade die einleitenden Kommentare zur Erzählung werden deshalb im Fokus dieses Beitrags stehen.

Der Forschung entging diese Vorrede zwar nicht, jedoch machten viele Analysen, die das Thema Geschichtsschreibung behandeln, zu schnell plakative Gegensatzpaare auf, um den Unterschied zwischen historiographischen und poetischen Erzählweisen hervorzuheben. Dass es demgegenüber interessant ist, die Gemeinsamkeiten ihrer narrativ-fiktionalen Erzählweisen in programmatischer Hinsicht für diese Fallgeschichte in den Blick zu nehmen, hat bisher wenig Beachtung gefunden. Auch für Historiker*innen gilt schließlich, dass sie Ereignisse aus der Vergangenheit in einen Zusammenhang bringen, indem sie die überlieferten Bruchstücke narrativ in einer Geschichte anordnen, die sie ihren Rezipient*innen erzählen. Sie können und sollen sich narrativ-fiktionalen Erzählweisen bedienen, betonen Daniel Fulda und Stefan Matuschek (201-204). Höcker bemerkt zu Recht, dass Erzähler*innen von Fallgeschichten in der erzählerischen Ausgestaltung poetische und historiographische Erzählweisen verbinden (In Citation 62). Vor dem Hintergrund von Schillers pädagogischen Bestrebungen weisen viele Bemerkungen des Erzählers in der Vorrede einen erzieherischen Charakter auf, die ich als moralisch-pädagogisch bezeichne. Durch solche Erzählweisen lenkt er die Lektüre seiner Rezipient*innen. Die erzähltheoretischen Ansätze in der Forschung zu Der Verbrecher aus verlorener Ehre stellen diese Zusammenhänge zwischen historiographischem und 
focus on German Studies.

https://journals.uc.edu/index.php/fogs

focus

(ISSN 1076-5697)

on German Studies

poetischem Erzählen auf der einen und den moralisch-pädagogischen Bemerkungen des Erzählers auf der anderen Seite bisher noch nicht deutlich genug heraus.

\section{Ein ,realer` Autor kommentiert: Überlegungen zur Historiographie}

Den nachfolgenden Ausführungen ist voranzustellen, wie das Verhältnis von Erzählendem und Erzähltem in der Vorrede von Der Verbrecher aus verlorener Ehre bestimmt ist:

Von dieser Seite betrachtet läßt sich manches gegen die gewöhnliche Behandlung der Geschichte einwenden, und hier, vermute ich, liegt auch die Schwierigkeit, warum das Studium derselben für das bürgerliche Leben noch immer so fruchtlos geblieben. (Schiller 13-14)

Weil der Erzähler in der Ich-Form erzählt, würden Forschende zunächst vermuten, dass das erzählende und das erzählte Subjekt identisch sind. Schaut man jedoch den Inhalt der Vorrede genauer an, dann wird klar, dass die Vorrede den rahmenden Teil der darauffolgenden Er-Erzählung bildet (Avgerinou 9): „Ob der Verbrecher, von dem ich jetzt sprechen werde, auch noch ein Recht gehabt hätte, an jenen Geist der Duldung zu appellieren?“ (Schiller 15). Nichtsdestoweniger referiert dieser Ich-Erzähler auf den empirischen Autor Friedrich Schiller. Der Erzähler ist also eine fiktionalisierte Figur, die die Autor*innenfunktion mit Namen ,Schiller repräsentiert. Durch die Stimme dieses Ich-Erzählers stellt Schiller seine allgemeinen Betrachtungen, die mehrere Diskurse zugleich in den Blick nehmen, der eigentlichen Geschichte über Christian Wolf voran. „[G]egen die gewöhnliche Behandlung der Geschichte“ wendet sich sogleich der erste Satz des Textes: „In der ganzen Geschichte des Menschen ist kein Kapitel unterrichtender für Herz und Geist als die Annalen seiner Verirrungen“ (Schiller 13). Der Erzähler merkt mit der Formulierung „Annalen seiner Verirrungen“ also gleich zu Beginn an, dass die „gewöhnliche Behandlung der Geschichte“ große Lücken aufweise. Mit den menschlichen „Verirrungen“ meint er die (in Vergessenheit geratenen) Geschichten über Verbrecher*innen, Missetäter*innen und Verirrte, die er gerade deshalb zum Thema macht. Diese Lücken zu 
focus on German Studies. https://journals.uc.edu/index.php/fogs (ISSN 1076-5697)

füllen sei für das Verstehen menschlicher Handlungen grundlegend. Indem der Erzähler die Begriffe „Herz“ und „Geist“ als Adressat*innen der ,unterrichtende[n]“ Maßnahmen beschreibt, weist er vor allem auf die von den „erkalte[ten]“ (Schiller 14) historiographischen und juristischen Erzählweisen wenig beachtete emotionale Struktur des Menschen hin:

Wenn sich das geheime Spiel der Begehrungskraft bei dem matteren Licht gewöhnlicher Affekte versteckt, so wird es im Zustand gewaltsamer Leidenschaft desto hervorspringender, kolossalischer, lauter[.] (Schiller 13)

Hier spricht der Erzähler bereits von der ,verhältnismäßig große[n] Kraft“ (Schiller 13) emotionaler Einflussnahme durch das „,geheime Spiel der Begehrungskraft“, die bei ,jedem großen Verbrechen“ (Schiller 13) in Erscheinung trete. Zur Entstehungszeit des Texts - das hat die Forschung klargestellt - beurteilte die Rechtsprechung ein begangenes Verbrechen auf der einen Seite strikt nach gesetzlichen Normen und auf der anderen Seite lediglich vom Standpunkt des verbrecherischen Aktes her. Wichtige Kontexte, wie beispielsweise die Lebensgeschichte des*der Verbrecher[s]*in, blieben unbeachtet: „Warum achtet man nicht in eben dem Grade auf die Beschaffenheit und Stellung der Dinge, welche einen solchen Menschen umgaben, bis der gesammelte Zunder in seinem Inwendigen Feuer fing? (Schiller 15). Der Erzähler bezieht hier emphatisch Stellung zu seiner Erzählweise, die die nachfolgende Fallgeschichte des Verbrechers Christian Wolf nicht nur im Hergang der Tat (re-)konstruieren, sondern zudem die Kontexte beleuchten will, die „einen solchen Menschen umgaben“ und ihn zu seinen verbrecherischen Akten trieben. Eine solche Erzählweise erweitert den historiographischen Ansatz, weil sie mit dem „Inwendigen“ einer Person eine Kategorie einführt, die selten in den überlieferten Quellen belegt ist: „An seinen Gedanken liegt uns unendlich mehr als an seinen Taten, und noch weit mehr an den Quellen seiner Gedanken als an den Folgen jener Taten“ (Schiller 15). Mit dem Fokus auf den „Zunder in seinem Inwendigen“ betont der Erzähler, dass ihn an der Lebensgeschichte Wolfs „das anthropologische Modell einer wahren Geschichte des 
focus on German Studies. https://journals.uc.edu/index.php/fogs (ISSN 1076-5697) focus on German Studies

Menschen“ (Lehmann, Im Abgrund der Wut 208) interessiert und - so Lehmann weiter - „das paradoxe Begehren nach Einschluss und Autonomie vor dem Hintergrund des Ausschlusses“(208). In diesem Verhältnis zwischen spezifischem Fall und allgemeinen Erkenntnissen, die die Rezipient*innen daraus ableiten sollen, bestätigen sich die eingangs erwähnten Überlegungen zur Erzählstruktur von Fallgeschichten. Leistet Der Verbrecher aus verlorener Ehre aber auch, was Rezipient*innen von einem historiographischen Text erwarten, dass er also, wie Ansgar Nünning festgehalten hat, das vergangene Geschehen (in diesem Fall: die Lebensgeschichte einer fiktiven Figur, die auf eine historische Person referiert) möglichst wirklichkeitsgetreu darstellt (366)?

Die bisherige Forschung beantwortete auch diese Frage mit einem klaren ,Nein'. Zwar liegt es auf der Hand, dass die Figur Christian Wolf auf den historischen Räuber Friedrich Schwan rekurriert. Allerdings war spätestens nach dem Erscheinen von Jacob Friedrich Abels (Re-)Konstruktion von Schwans Geschichte (Abel) klar, dass Schillers ein Jahr zuvor veröffentlichte Version in vielerlei Hinsicht erfunden ist. Schiller leistete aber nicht etwa mangelhafte historiographische Arbeit, sondern entschied sich bewusst für eine Schreibweise, die den historischen Fall von mehreren Seiten aufschlüsselt. Forscher*innen wie Lüdemann leiten daraus zweierlei ab: Es sei für Schiller erstens nicht möglich gewesen, faktische Belege für einige in Der Verbrecher aus verlorener Ehre angesprochene Themen aus den überlieferten Quellen zu erhalten. Zweitens meine Schiller - obwohl er seine Erzählung eine „wahre Geschichte“ nennt - nicht (nur) die faktische Wahrheit des vergangenen Geschehens, sondern eine ,poetische Wahrheit‘. Sie gehe über die bloßen historischen Fakten hinaus, weil sie von größerer Allgemeinheit sei und die ihr zugrundeliegenden Fallgeschichten über individuelle menschliche Schicksale anders erzähle, zum Beispiel, indem sie die Innensicht der behandelten Figuren schildere (Lüdemann 216). Poetische Texte können diese in historiographischen Texten zumeist als ,dark areas‘ verbleibenden Aspekte besser beleuchten und somit erkenntnisstiftend wirken. Dazu 
focus on German Studies. https://journals.uc.edu/index.php/fogs (ISSN 1076-5697) focus on German Studies

bedarf es aber eines gewissen Maßes an emotionaler Bereitschaft auf Seiten der Rezipient*innen, sich auf das, was der*die Erzähler*in präsentiert, einzulassen.

Dieses Problem wird bereits in der Vorrede deutlich:

Zwischen der heftigen Gemütsbewegung des handelnden Menschen und der ruhigen Stimmung des Lesers, welchem diese Handlung vorgelegt wird, herrscht ein so widriger Kontrast, liegt ein so breiter Zwischenraum, dass es dem letztern schwer, ja unmöglich wird, einen Zusammenhang nur zu ahnden.

Es bleibt eine Lücke zwischen dem historischen Subjekt und dem Leser, die alle Möglichkeit einer Vergleichung oder Anwendung abschneidet und statt jenes heilsamen Schreckens, der die stolze Gesundheit warnet, ein Kopfschütteln der Befremdung erweckt. (Schiller 14)

Indem der Erzähler einen „widrige[n] Kontrast“ zwischen der „heftigen Gemütsbewegung“ einer erzählten Figur und „der ruhigen Stimmung“ der Rezipient*innen aufmacht, bereitet er die Leser*innen auf die nachfolgende Er-Erzählung vor. „[H]eftige[ Gemütsbewegung[en]“ gibt es schließlich nicht ohne Grund, und wenn der Erzähler in seiner Erzählung die Geschichte des fiktiven Verbrechers Christian Wolf durchleuchtet, will er zuvorderst darstellen, wie Wolf zum Verbrecher wurde. Im selben Zuge unterstellt er seinen Rezipient*innen eine „ruhige[ Stimmung“ beim Lesen, ganz so, als wäre das die einzige Art, wie ein Mensch gegen Ende des 18. Jahrhunderts liest. Er benötigt aber beides, den von vielerlei Emotionen getriebenen Protagonisten und die ruhig gestimmten Leser*innen, damit seine Erzählweise aufgeht. Denn dem Erzähler zufolge können juristische oder historiographische Erzählweisen ihren Adressat*innen die Vermittlung des für ihn so wichtigen „Zusammenhang[s]“ zwischen einem Verbrechen und seinen Ursachen nicht leisten:

Wir sehen den Unglücklichen, der doch in eben der Stunde, wo er die Tat beging, so wie in der, wo er dafür büßet, Mensch war wie wir, für ein Geschöpf fremder Gattung an, dessen Blut anders umläuft als das unsrige, dessen Wille andern Regeln gehorcht als der unsrige[.] (Schiller 14) 
focus on German Studies. https://journals.uc.edu/index.php/fogs (ISSN 1076-5697) focus on German Studies

Mit dem Wechsel des Fokus hin zu den Hintergründen eines Verbrechens und der Innensicht der*des Verbrecher $[\mathrm{s}] *_{\text {in }}$ verstehen die Rezipient*innen nicht nur ein Verbrechen und dessen Akteur*innen besser. Mit besserem Verständnis fällt auch nach und nach die vom Erzähler so sehr bemängelte „Lücke zwischen dem historischen Subjekt und dem Leser“" weg. Infolge dessen akzeptieren die Leser*innen die Verbrecherfiguren, weil sie - so auch Susanne Kaul - ähnliche menschliche Verhaltensweisen wie sie selbst aufweisen (239): Sie sehen sie nicht mehr als „Geschöpf[e] fremder Gattung“ an. Damit stellt der Erzähler die Weichen für die „Möglichkeit einer Vergleichung“ sowie der emotionalen Teilnahme, nämlich insofern er von seiner poetischen Erzählung behauptet, sie beleuchte die im Vergleich zu historiographischen oder juristischen Texten unbeachteten ,dark areas': „[S]eine Schicksale rühren uns wenig, denn Rührung gründet sich ja nur auf ein dunkles Bewußtsein ähnlicher Gefahr“ (Schiller 14). Mit der autodiegetischen Fokalisierung verleiht der Erzähler dem Verbrecher Wolf eine Stimme, die ausdrückt, was menschlich ist, die jede*r Rezipient*in nachvollziehen kann und die somit das „dunkle[ Bewußtsein ähnlicher Gefahr“ fördert. Ich stimme deshalb Philipp Webers These, dass es sich in der Vorrede um eine „die Stimme der Wissenschaft imitierende“ (307), „neutrale Erzählstimme“ (309) handele, nicht zu. Lüdemann zufolge beglaubigen die Leser*innen, wenn sie Fallgeschichten derart nachvollziehen, „die Allgemeinheit der Darstellung als Allgemeinheit des Dargestellten“ (217). Mit Yvonne Nilges lässt sich dieses Erzählverfahren durchaus als eines bezeichnen, das teilweise im Dienste einer kriminellen Ätiologie steht (22).

Hinter all dem steht schließlich die moralische Belehrung: „Die Belehrung geht mit der Beziehung verloren, und die Geschichte, anstatt eine Schule der Bildung zu sein, muß sich mit einem armseligen Verdienste um unsre Neugier begnügen“ (Schiller 14). Wenn die Rezipient*innen es nicht schaffen, eine „Beziehung“ zu den Personen, über die sie lesen, herzustellen, verfehle ein historiographischer Text seine für den Erzähler so wichtige belehrende Funktion. Mit diesem radikalen Urteil ist ein ,nur ' historiographischer Text im Grunde genommen nicht mehr viel wert. Aufgrund der zu großen Distanz „zwischen dem 
focus on German Studies. https://journals.uc.edu/index.php/fogs (ISSN 1076-5697) focus on German Studies

historischen Subjekt und dem Leser“ sei die Lehre, die Rezipient*innen aus diesen Texten ziehen könnten, unerreichbar. Nicht einmal die erste Hürde hin zur Erkenntnis sei durchbrochen, wenn sich Historiker*innen lediglich um den „armseligen Verdienste um unsre Neugier“ kümmerten:

Soll sie uns mehr sein und ihren großen Endzweck erreichen, so muß sie notwendig unter diesen beiden Methoden wählen - Entweder der Leser muß warm werden wie der Held, oder der Held wie der Leser erkalten. (Schiller 14)

Dieser Lösungsvorschlag des Erzählers ist Thema vieler Untersuchungen. Achim Geisenhanslüke resümiert in seinem Beitrag, dass der Erzähler seine ,prosaische Darstellung des Verbrechens an den kalten Blick [...] binde $\rrbracket$, der den Dichter und den Historiker in diesem Fall verbinde [“ (126). Die Gemeinsamkeiten beider, also der poetischen und historiographischen, Erzählweisen kommen demnach deshalb zustande, weil die poetische Erzählweise die historiographische bis zu einem gewissen Grad nachahmt. Geisenhanslüke führt dies auf den „prosaischen Gegenstand des Verbrechens“(216) zurück, den der Erzähler nur über die „erkalte[te]“ Methode der Historiker*innen angemessen beschreiben könne. Die poetische Erzählweise muss sich also - soweit vermittelt das die Rede des Erzählers bis hierhin - weiterentwickeln und ein*e Erzähler*in soll sie insbesondere mit historiographischen Verfahren anreichern. Das würde jedoch bedeuten, dass sich der Erzähler mit historischen Quellen auseinandersetzen müsse. Gerade das leistet die Erzählung aber nicht.

Verblüffend erscheint sodann die darauffolgende Passage:

Ich weiß, daß von den besten Geschichtsschreibern neuerer Zeit und des Altertums manche sich an die erste Methode gehalten und das Herz ihres Lesers durch hinreißenden Vortrag bestochen haben. Aber diese Manier ist eine Usurpation des Schriftstellers und beleidigt die republikanische Freiheit des lesenden Publikums, dem es zukömmt, selbst zu Gericht zu sitzen[.] (Schiller 14)

Einen Satz zuvor plädiert der Erzähler dafür, dass die poetische Erzählweise historiographische Erzählweisen imitieren solle. Hier urteilt der Erzähler in größter Abwehrhaltung den umgekehrten Fall ab. Es stehe einer 
focus on German Studies. https://journals.uc.edu/index.php/fogs (ISSN 1076-5697) focus on German Studies

historiographischen Erzählweise nicht zu, sich als poetische auszugeben und „das Herz ihres Lesers durch hinreißenden Vortrag“ zu bestechen. Mehr noch: Wenn Historiker*innen poetisch erzählen, beanspruchen sie eine Erzählweise, die nur den Schriftsteller*innen rechtmäßig zustehe und zudem kränken sie die Rezipient*innen, deren Lektüre von historiographischen Texten sie nicht beeinflussen sollen. Eine klare Grenzziehung zwischen den unterschiedlichen Erzählweisen von Historiographie und Poetik macht der Erzähler den Leser*innen damit deutlich: poetisch in historiographischen Texten zu erzählen sei „eine Verletzung der Grenzengerechtigkeit, denn diese Methode gehört ausschließend und eigentümlich dem Redner und Dichter. Dem Geschichtsschreiber bleibt nur die letztere übrig“ (Schiller 14). Es ist bemerkenswert, mit welcher Selbstverständlichkeit der Erzähler diese widersprüchlichen Redeweisen einander gegenüberstellt, begründet er doch zu keinem Zeitpunkt, warum lediglich die poetische Erzählweise das „Herz“ ihrer Adressat*innen bestechen darf. Dies kulminiert darin, dass er die eigens heraufbeschworene „Grenzengerechtigkeit“ immer dann selbst durchbricht, wenn er die stets zu erweiternden Möglichkeiten poetischer Erzählweisen, beispielsweise durch den Einbezug historiographischer Verfahren, legitimieren will: „Der Held muß kalt werden wie der Leser, oder [...] wir müssen mit ihm bekannt werden, eh er handelt, wir müssen ihn seine Handlung nicht bloß vollbringen, sondern auch wollen sehen“ (Schiller 14-15). Entzieht der Erzähler seinen Rezipient*innen nicht gerade auch dort, wo er poetisch erzählt, die Möglichkeit, ,,selbst zu Gericht zu sitzen“? Warum müssen Leser*innen denn die „Handlung [Wolfs] nicht bloß vollbringen, sondern auch [...] sehen" wollen? Durch den Vergleich zwischen poetischen und historiographischen Erzählweisen gelingt es dem Erzähler vor allem, seine poetische als die einzige Erzählweise darzustellen, die die Fallgeschichte Wolfs allumfassend erschließen kann. Sie kritisiert nicht nur die vermeintlich eindeutigen Geschichten historiographischer Texte, sondern erzählt den Fall Wolfs durch seine vielfältigen und zugleich gleichrangigen Perspektiven mehrdeutig und unabgeschlossen. 
focus on German Studies.

https://journals.uc.edu/index.php/fogs

focus

(ISSN 1076-5697)

on German Studies

\section{Moralisch-pädagogische Bemerkungen: Leser*innenlenkung und Mehrdeutigkeit}

Viele Forschungsbeiträge beachteten, wie Steffen Martus zu Recht konstatiert, diese widersprüchlichen Ankündigungen in der Vorrede für das nachfolgende Erzählprogramm nicht (255 und 264). Auch Jeffrey L. High betont, dass der Erzähler nicht die Absicht verfolge, seinen Helden erkalten zu lassen. Vielmehr verweise er auf die „erkalte[te]“ Erzählweise einer historiographischen oder juristischen Darstellung durchaus absichtlich, um seine eigene überhaupt nicht „erkalte[te]“ moralisch-pädagogische Erzählhaltung zu legitimieren (177). Er will den historischen Bezug schließlich nicht verlieren, weil er durch ihn seine moralische Botschaft vermitteln kann. Das „anthropologische Modell einer wahren Geschichte des Menschen“ (Lehmann, Im Abgrund der Wut 208) macht er demnach als „Freund der Wahrheit“ (Schiller 15) für seine Rezipient*innen relevant, und zwar, indem er den Menschen bzw. die „Struktur der menschlichen Seele“ (Schiller 15) im Zusammenspiel mit der menschlichen Lebenswelt betrachtet:

Er [der Freund der Wahrheit] sucht sie in der unveränderlichen Struktur der menschlichen Seele und in den veränderlichen Bedingungen, welche sie von außen bestimmten, und in diesen beiden findet er sie gewiß. (Schiller 15)

Die „Struktur der menschlichen Seele“ lässt sich nichts zuschulden kommen, weil sie „unveränderlich[“ sei. Veränderlich seien lediglich die „Bedingungen, welche sie von außen be-stimmten“. Ohne genauer darauf einzugehen, weshalb das so ist, versucht der Erzähler im weiteren Verlauf immer stärker die Sichtweise seiner Rezipient*innen zu lenken. Wenn er die Ursachen für Wolfs Verbrechen zum Thema seiner Erzählung macht und gleichzeitig sagt, dass sie von „außen“ kämen, erklärt er seine Rezipient*innen nicht nur - wie Achim Aurnhammer in seinem viel zitierten Beitrag folgert - zu „Verbündeten seines Engagements gegen die herrschenden Normen und für eine humane Gesellschaft“ (259). Wie neben anderen Pfeiffer feststellt, steuert er den Leseakt geradezu und macht noch vor Beginn der Binnenerzählung nicht wenige und zum Teil widersprüchliche Vorgaben für die Lektüre der Geschichte über Christian Wolf (110): ,[O]b er wirklich ohne 
focus on German Studies. https://journals.uc.edu/index.php/fogs (ISSN 1076-5697) focus on German Studies

Rettung für den Körper des Staats verloren war“ (Schiller 15)? Das ist einerseits verwirrend und zweifellos manipulativ; andererseits verdeutlicht es aber, dass er seine moralisch-pädagogischen Bemerkungen in einer, wie Avgerinou treffend beschreibt, „ambivalenten Haltung“(15) zum Ausdruck bringt: „Ich will dem Ausspruch des Lesers nicht vorgreifen“ (Schiller 15). Was will er mit diesen wiederholt widersprüchlichen Reden bezwecken?

Dem Erzähler liegt viel daran, die Vermittlung seiner moralischen Botschaft gut zu begründen: „[A]ber die Leichenöffnung seines [Wolfs] Lasters unterrichtet vielleicht die Menschheit und - es ist möglich, auch die Gerechtigkeit“ (Schiller 15). Diese Bemerkung lenkt die Leser*innen nicht nur in negativem Sinne, sondern wirkt auch positiv auf sie ein. Sie erzieht zu einer - um die Relevanz von Der Verbrecher aus verlorener Ehre durch einen aktuell oft diskutierten Begriff des Islamwissenschaftlers Thomas Bauer hervorzuheben „ambiguitätstolerant[en]“ (16) Haltung. Eine solche Haltung ermöglicht es ihren Träger*innen, dass sie es aushalten, die Welt mehrdeutig zu erklären bzw. erklärt zu bekommen. Die daraus entstehenden Widersprüche erachtet man nicht als sinnlos, sondern akzeptiert sie als menschliche Gegebenheit, wenn nicht gar Voraussetzung (Bauer 79). Das mag zwar eine These sein, die man, weil sie modern gedacht ist, nicht so ohne Weiteres auf das Weltbild zu Schillers Zeit übertragen kann. Ihre Plausibilisierung würde aber bestätigen, dass Der Verbrecher aus verlorener Ehre - einmal abgesehen von seiner Kanonisierung - zeitlos ist und gerade in unserer gegenwärtig ausdifferenzierten Gesellschaft wichtige Voraussetzungen für das Gelingen von gesellschaftlichem Zusammenhalt vermittelt. Martus spricht dem Text daher nicht ohne Grund zu, dass er seine Rezipient*innen zu „selbstläuferisch[en] und [...] sozialverträglich[en]“ (269) Menschen ausbilde. Durch welche Textstellen lässt sich nun aber diese These belegen? Relativiert oder legitimiert die Erziehung zu einer ambiguitätstoleranten Haltung sogar den manipulativen Charakter der Rede des Erzählers?

Um eine Antwort zu finden, lohnt es sich, zunächst einen Überblick über die von der Forschung bereits herausgearbeitete narrative Struktur im Verbrecher aus verlorener Ehre zu geben. Markus Biesdorf etwa 
focus on German Studies.

https://journals.uc.edu/index.php/fogs

focus

(ISSN 1076-5697)

on German Studies

hält fest, dass verschiedene Teile des Texts unterschiedlich fokalisiert sind. Durch die unterschiedlichen

Fokalisierungen könnten Rezipient*innen die Fallgeschichte Wolfs aus verschiedenen Blickwinkeln betrachten (286). Damit setzt der Erzähler seine in der Vorrede angekündigte ,ambivalente[ Haltung“ (Avgerinou 15) um. Avgerinou stellt in ihrer narratologischen Betrachtung die Nuancen dieser Erzählstruktur bis ins kleinste Detail heraus:

In der Erzählung sind zwei narrative Ebenen unterscheidbar. Die erste konstituiert der Erzählakt des Er-Erzählers. Die Ich-Erzählung stellt auf den ersten Blick nur eine Fortführung der Narration dar; [...] der Erzähler Wolf nimmt das Wort und führt seine Geschichte weiter gerade vom Punkt, wo der Er-Erzähler ihm das Wort übergeben hat. Dennoch erfüllen sich in diesem Fall alle Kriterien, die nach Genette die Existenz einer anderen, zweiten narrativen Ebene nahelegen. Die Ich-Erzählung ist nämlich in der Erzählung des Er-Erzählers enthalten [...] der Erzähler berichtet, was ein anderer vor ihm berichtet hat. [...] Auch ist der Erzähler der Ich-Erzählung bereits eine Figur der ersten, der ErErzählung. Also ist der Er-Erzähler, die narrative Instanz der ersten Erzählung, extradiegetisch in Bezug auf die zweite, metadiegetische Erzählung, während der Ich-Erzähler eine diegetische narrative Instanz konstituiert. (8-9)

In der auf die Vorrede folgende „Er-Erzählung herrscht also eine Nullfokalisierung [...] vor, während die IchErzählung intern fokussiert ist“ (Avgerinou 8). Und weiter: „Heterodiegetisch ist Schillers Geschichte bei der Er-Erzählung [...] Die Ich-Erzählung im Verbrecher ist dagegen homodiegetisch [...] und zwar autodiegetisch, da Wolf die Hauptfigur ist“ (Avgerinou 9). Übersetzt in das narratologische Vokabular Gérard Genettes erscheint diese Erzählweise vielschichtig und auch durchaus verwirrend. Dass sie die Fallgeschichte Wolfs hingegen gerade durch die ständigen Wechsel der Erzählebenen, Fokalisierungen und narrativen Modi mehrdeutig erzählt und ihre widersprüchlichen Reden dabei trotzdem angemessen verständlich wiedergibt, ist die größte Errungenschaft dieser Erzählung. 
focus on German Studies.

https://journals.uc.edu/index.php/fogs

(ISSN 1076-5697) focus

on German Studies

Die komplexen Ergebnisse der narratologischen Analyse dürfen deshalb nicht irritieren, lassen sich doch auch sie weiterdenken. Avgerinou leistet das und betont, dass „die narrative Pause am Anfang der Erzählung und der dramatische Schluss“(20) erzählerische Arrangements seien, die ihre Rezipient*innen absichtlich mit Widersprüchen konfrontierten. Bangert und Müller-Richter heben zudem hervor, dass diese Momente der Irritation wichtig seien, um einer eindeutigen Erschließung der Reden der Erzähler entgegen zu wirken (282). Obwohl Martus diese erzählerischen Gestaltungsmittel wahrscheinlich eher Schillers Absicht zuordnen würde, publikumswirksam zu schreiben, verschweigt er nicht, dass die Erzählinstanzen in Der Verbrecher aus verlorener Ehre

in der Beschäftigung mit dem im Detail Interessanten eine Beobachtungshaltung [erzeugen], die für eine komplexe, schwer oder gar nicht zu verstehende Welt bereit ist, die mit hohen Anforderungen an Deutungskompetenz zurechtkommt und die Unschärfen bei der Zurechnung von Verantwortlichkeit erträgt. (269)

Bemerkenswert ist zudem, dass die Forschung zwar häufiger den Zusammenhang zwischen den Beschreibungen in Michel Foucaults Überwachen und Strafen und Schillers Erzählung herstellt, aber eben nicht in narratologischer Hinsicht. Foucault schreibt:

Welcher Ebene oder welchem Bereich ist die Tat zuzuordnen - Wahngebilde, psychotische Reaktion, Augenblick der Verwirrung, Perversität? Nicht mehr einfach: ,Wer ist der Täter?‘, sondern: ,Wie kann man den Kausalprozeß, der zur Tat geführt hat, einordnen? Wo ist sein Ursprung im Täter selbst? Instinkt, Unbewußtes, Milieu, Erbanlage?’ Nicht mehr einfach: ,Welches Gesetz sanktioniert diese Vergehen?‘, sondern: ,Welche Maßnahmen sind die angemessensten? (29)

Gerade den von Foucault angesprochenen „Kausalprozeß“ setzt der Erzähler der Vorrede erzählerisch um, beginnend mit der Vorstellung Wolfs und seinem schweren Lebensweg: „Vater tot, missliche finanzielle Situation [...], abstoßendes Aussehen“ (Kaul 242). Hinzu kommt, dass ihn das „Mädchen, das er wählte, 
focus on German Studies. https://journals.uc.edu/index.php/fogs (ISSN 1076-5697) focus on German Studies

mißhandelte“ (Schiller 16) und sein Nebenbuhler Robert ihn während seiner Wilddiebstähle zweimal ergreift, sodass man ihn letztlich ,in das Zuchthaus der Residenz“ (Schiller 17) abliefert. Bereits vor der zweiten Festnahme geselle sich in Wolf ein „[d]rückendes Gefühl des Mangels [...] zu beleidigtem Stolze, Not und Eifersucht stürmen vereinigt auf seine Empfindlichkeit ein [...], Rache und Leidenschaft halten ihn fest"“ (Schiller 17). Nachdem er sein „Strafjahr“ (Schiller 17) hinter sich gebracht hat, meiden ihn die Menschen in seiner Heimat und er wird, arbeitssuchend, „an allen Orten zurückgewiesen“ (Schiller 17). Seine prekäre Lage zwingt ihn dazu, zum dritten Mal einen Wilddiebstahl zu begehen und ,zum drittenmal trifft ihn das Unglück, seinem wachsamen Feind in die Hände zu fallen“ (Schiller 17). Die Richter verurteilen Wolf daraufhin zu einer dreijährigen Festungshaft, so der Erzähler, bevor er die autodiegetische Erzählung Wolfs beginnen lässt: „[M]an höre ihn selbst, wie er nachher gegen seinen geistlichen Beistand und vor Gerichte bekannt hat" (Schiller 18). Mit seiner Anmerkung macht der Erzähler den Leser*innen deutlich, dass er diese ,Vorgeschichte aus dem Bericht Wolfs vor dem Gericht übernommen und für sie zusammengefasst hat. Das ist durchaus ein wichtiges Detail, da es auf die erzählerische Umsetzung sowohl des von Foucault beschriebenen Kausalprozesses als auch der in der Vorrede erhobenen Forderung, den Helden wie den Leser erkalten zu lassen, hindeutet. Schließlich hat der Erzähler die Hintergrundinformationen über den Werdegang Wolfs aus dessen Bericht vor Gericht entnommen und somit zumindest in der erzählten Welt Quellenarbeit im historiographischen Sinne geleistet. Der vorwiegend nüchterne und sachliche Ton dieser Vorgeschichte ist daher auch nicht dem Erzähler, sondern Wolf selbst zuzuschreiben. Vor Gericht stand also ein sachlich argumentierender Mensch, der den Kausalprozess, welcher zu seinen verbrecherischen Taten geführt hatte, präzise darstellen konnte. Die Schuld an seinen Taten trägt somit nicht nur er selbst. Ganz im Sinne Foucaults tragen auch die „Disziplinargesellschaften [des] 18. und 19. Jahrhundert[s]“ (Deleuze 254), die ihn nicht wieder eingliedern, maßgeblich zu seinem gesellschaftlichen Fall bzw. Abstieg bei. Insofern löst der Erzähler auch 
focus on German Studies.

seine Forderung, dass die Leser*innen „mit ihm bekannt werden [sollen], eh er handelt“ (Schiller 14), erzählerisch ein.

All diese Erzählweisen spiegeln die ambiguitätstolerante Haltung des Erzählers wider. Bereits im zweiten Abschnitt der Vorrede kommt er auf sie zu sprechen:

Es ist etwas so Einförmiges und doch wieder so Zusammengesetztes, das menschliche Herz. Eine und eben dieselbe Fertigkeit oder Begierde kann in tausenderlei Formen und Richtungen spielen, kann tausend widersprechende Phänomene bewirken, kann in tausend Charakteren anders gemischt erscheinen, und tausend ungleiche Charaktere und Handlungen können wieder aus einerlei Neigung gesponnen sein, wenn auch der Mensch, von welchem die Rede ist, nichts weniger denn eine solche Verwandtschaft ahndet. (Schiller 13)

Mit dieser Aussage deutet der Erzähler an, dass das „,menschliche Herz“, womit er auf die innere bzw. psychische Struktur/Verfassung des Menschen anspielt, „in tausenderlei Formen und Richtungen spiele[“. Er gibt also schon an dieser Stelle vor, auf was sich die Leser*innen beim Lesen seiner Fallgeschichte einlassen werden. Sie sollen ihre menschliche „Verwandtschaft“ mit einer (fiktiven) Verbrecherfigur nicht nur „ahnde[n]“, sondern sie verstehen. Menschlich zu sein hieße hier, dass unterschiedliche und sogar gleiche Lebensumstände in „tausend Charakteren anders gemischt erscheinen.“ Die Kontexte, die Wolf zum Verbrecher machen, können andere wiederum zu rechtmäßig handelnden Individuen machen:

[W]ie sehr würde man erstaunen, wenn man so manchen, dessen Laster in einer engen bürgerlichen Sphäre und in der schmalen Umzäunung der Gesetze jetzt ersticken muß, mit dem Ungeheuer Borgia in einer Ordnung beisammen fände. (Schiller 13)

Beide Entwicklungen begründet und legitimiert er aus der „eine[n] Ordnung“ heraus, die alle Menschen beeinflusse: die Gesellschaft, in der sie aufwachsen und leben. Für alle Menschen gelte somit, individuell 
focus on German Studies. https://journals.uc.edu/index.php/fogs (ISSN 1076-5697)

„,z]usammengesetzte[ [...] Herz[en]“ zu besitzen, die „tausend ungleiche Charaktere und Handlungen“ hervorbringen.

Mit solchen Aussagen will der Erzähler zweierlei erreichen: Zum einen will er festgefahrene „gesellschaftliche】 Normierungs- und Normalisierungsprozesse“ (Lüdemann 212) durch neue Erkenntnisse der „Seelenkunde“ (Schiller 15) kritisieren und reflektieren:

Wenn ich auch keinen der Vorteile hier in Anschlag bringe, welche die Seelenkunde aus einer solchen Behandlungsart der Geschichte zieht, so behält sie schon allein darum den Vorzug, weil sie den grausamen Hohn und die stolze Sicherheit ausrottet, womit gemeiniglich die ungeprüfte aufrechtstehende Tugend auf die gefallne herunterblickt, weil sie den sanften Geist der Duldung verbreitet, ohne welchen [...] keine Aussöhnung des Gesetzes mit seinem Beleidiger stattfindet, kein angestecktes Glied der Gesellschaft von dem gänzlichen Brande gerettet wird. (Schiller 15)

Zum anderen erklärt er seine poetische, wiederum im Gegensatz zur ,üblichen“ „Behandlungsart der Geschichte“, zu einer Erzählweise, die den Fall Wolfs in all seinen Facetten aufarbeite. Denn sie bringt die Empathie oder nach Kaul, „Sympathie [...], Mitleid und Verständnis“ (249) für den Verbrecher mit einer emphatischen Vortragsweise zusammen und kämpft damit gegen „den grausamen Hohn und die stolze Sicherheit“ an, die die Menschen ergreife, sobald sie einem Geächteten gegenüberständen. Heraus kommt eine ambiguitätstolerante Haltung in Form eines „sanften Geist[es] der Duldung“, der zudem die „Lücke zwischen dem historischen Subjekt und dem Leser“ verringere. Agnieszka Magdalena Rybska bemerkt allerdings, dass solche Ausführungen den Verbrecher Wolf, der zweifellos schuldig ist, aufgrund seines tragischen Schicksals für die Leser*innen eher als unschuldig erscheinen lassen (52). Auffällig ist vor allem ein Cut auf der histoire-Ebene, mit dem der Erzähler die Ich-Erzählung Wolfs beendet und wiederum zur ErErzählung überleitet: 
focus on German Studies. https://journals.uc.edu/index.php/fogs

(ISSN 1076-5697) focus

on German Studies

Den folgenden Teil der Geschichte übergehe ich ganz, das bloß Abscheuliche hat nichts Unterrichtendes für den Leser. Ein Unglücklicher, der bis zu dieser Tiefe heruntersank, mußte sich endlich alles erlauben, was die Menschheit empört - aber einen zweiten Mord beging er nicht mehr, wie er selbst auf der Folter bezeugte. (Schiller 28-29)

Rybska folgert richtig, dass der Erzähler hier zu einer Ellipse greift, um seine moralisch-pädagogischen Bemerkungen nicht selbst zu unterlaufen. Das „bloß Abscheuliche“ würde die Rezipient*innen schließlich zu „einer ausschließlich negativen Beurteilung von Wolf veranlassen“ (49-50). Für Historiker*innen würde ein solches Verfahren, nebenbei angemerkt, einen Verstoß gegen die wissenschaftlichen Richtlinien ihres Fachs bedeuten. Zugleich legitimiert er das „bloß Abscheuliche“ mit Wolfs unausweichlichem Fall in menschliche Abgründe und erwähnt zusätzlich noch, dass er keinen „zweiten Mord beging“. Mehr Verständnis kann ein Erzähler für seine fiktive Verbrecherfigur in zwei Sätzen kaum formulieren.

Die Forschung sollte vielleicht darüber streiten, ob die damit einhergehende, wenn auch berechtigte Kritik an der Gerechtigkeit der Rechtsprechung, die Wolf im Verlauf der Geschichte mehrmals bezweifelt, zu sehr in die Denkweise ihrer Empfänger*innen eingreift. Schließlich kommt es ihnen laut dem Erzähler zumindest, wenn sie historiographische Texte lesen - ja eigentlich zu, „selbst zu Gericht zu sitzen“ (Schiller 14). Dazu müsste die Forschung aber den historischen Kontext, insbesondere zum Stand der Bildung in der Bevölkerung, einbeziehen. Beispielsweise würden sie dann der Frage nachgehen, ob die Menschen zu Schillers Lebzeiten sowohl im öffentlichen als auch privaten Bereich überhaupt Kritik am Rechtssystem üben konnten.

Auch wenn der Erzähler seine Rezipient*innen massiv beeinflusst, kann man ihm jedoch nicht absprechen, dass er seine moralisch-pädagogischen Bemerkungen auf einen wünschenswerten Zweck richtet: Ihn überrascht es nun nicht mehr, in dem nämlichen Beete, wo sonst überall heilsame Kräuter blühen, auch den giftigen Schierling gedeihen zu sehen, Weisheit und Torheit, Laster und Tugend in einer Wiege beisammen zu finden. (Schiller 15) 
focus on German Studies. https://journals.uc.edu/index.php/fogs (ISSN 1076-5697) focus

on German Studies

Die durch die moralisch-pädagogischen Bemerkungen des Erzählers zu einer ambiguitätstoleranten Haltung ausgebildeten Adressat*innen erkennen bestenfalls genau das: „[...] in dem nämlichen Beete“, dem einer menschlichen Gesellschaft, wachsen nicht nur „heilsame Kräuter“, leben nicht nur systemkonforme Menschen, sondern auch ,giftige[ Schierling[e]“, also Menschen, die auf Abwege geraten und gesellschaftliche Normen brechen. Entscheidend ist dabei jetzt, dass trotz der widersprüchlichen Verhaltensweisen all diese mehrdeutigen Handlungsweisen ,in einer Wiege beisammen“, also durch den gesellschaftlichen Einfluss hervorgebracht werden. Das bedeutet, dass ein Mensch auf Abwegen sich jederzeit wieder resozialisieren kann - vorausgesetzt, er oder sie trifft auf eine ambiguitätstolerante Gesellschaft, die das zulässt, weil sie erkannt hat, dass „Weisheit und Torheit, Laster und Tugend in einer Wiege beisammen“ sind.

\section{Ausblick: Ein ertragreicher Text für narratologische Analysen}

Aus der Analyse geht hervor, dass der Erzähler in Der Verbrecher aus verlorener Ehre solche Texte kritisiert, die einen Fall lediglich von einer Seite betrachten. Im Gegensatz zu den eindeutigen Erzählweisen historiographischer Texte ist die poetische Erzählweise des Erzählers der Vorrede mit ihren moralischpädagogischen Bemerkungen dafür geeignet, Mehrdeutigkeit herzustellen. Mithilfe unterschiedlicher erzählerischer Gestaltungsmittel bringt er diese Kritik somit auch erzählerisch zum Ausdruck. Die zentrale Fragestellung, nämlich mit welchen Argumenten kritisiert der Erzähler der Vorrede in Der Verbrecher aus verlorener Ehre, dass bestimmte Erzählweisen einen Fall jeweils eindeutig erschließen - beantwortete ich nun folgendermaßen:

Meine erste These besagt, dass der Erzähler bei zugleich erfolgender Kritik an der und Distanzierung von der Geschichtsschreibung dennoch historiographische Verfahren für seine Poetik beansprucht. Dazu (re)konstruiert er die Fallgeschichte des Verbrechers Christian Wolf nicht nur in ihren Tathergängen, sondern beleuchtet insbesondere auch den Kausalprozess, der aufzeigt, welche Faktoren diese Figur zum Verbrecher 
focus on German Studies. https://journals.uc.edu/index.php/fogs (ISSN 1076-5697) focus on German Studies

gemacht haben. Entscheidend ist zudem das Innenleben seiner Figur. Damit begründet er, dass seine poetische Erzählung die im Vergleich zu historiographischen Erzählweisen unbeachteten ,dark areas` aufzeige. Diese wiederum unterstützen die Rezipient*innen dabei, die fiktive Figur Christian Wolf als nahbar zu erfahren. So bringen sie während der Lektüre Empathie für Wolf auf.

Vor dem Hintergrund, dass der Erzähler seine Rezipient*innen durch diese Erzählweise belehren will, ist meine zweite These, dass eine mehrdeutige Betrachtung des Verbrechers Wolf notwendig ist. Die narrative Struktur des Textes belegt dies: Durch ständige Wechsel der Erzählebenen, Fokalisierungen und narrativen Modi stellt der Erzähler die Fallgeschichte Wolfs mehrdeutig dar und versäumt dabei trotzdem nicht, die daraus entstehenden Widersprüche verständlich wiederzugeben. Im selben Zug erklärt er die eigene poetische zur einzigen Erzählweise, die den Fall Wolf in all seinen Facetten aufarbeiten könne. Ziel ist die Erziehung der Leser*innen zu einer ambiguitätstoleranten Haltung, mittels der sie die Taten Wolfs auf der einen Seite als menschliche Gegebenheiten akzeptieren und auf der anderen Seite als Produkt ihrer Gesellschaft betrachten.

Es gäbe noch viele Anhaltspunkte, die einen differenzierteren Blick auf die aufgestellten Thesen ermöglichen würden. Eine detaillierte Untersuchung der Binnenerzählungen ist mit Sicherheit der wichtigste Punkt. Dabei sollten sich Forschende an den Analysen Kauls (241-247) orientieren und herausarbeiten, wie der Erzähler die unterschiedlichen Erzählebenen erzählerisch gestaltet (welche Erzähler*innen setzt er an welchen Stellen ein? Wo ändert er narrative Modi und zu welchem Zweck?) und an welchen Stellen er seinem in der Vorrede angekündigten Erzählprogramm widerspricht. Zudem geben die Binnenerzählungen noch viele weitere Hinweise zur Leser*innenlenkung, so beispielsweise in einer Passage, in der Wolf seinen Fürsten in einem Brief um Begnadigung bittet. Mark-Georg Dehrmann schreibt dazu:

Der Sonnenwirt schreibt hier nicht als »anderes Wesen« aus einer »anderen Klasse«, mit fremdartigem Lebensstil. Er spricht vielmehr die vertraute Sprache des republikanischen Publikums. Schiller schafft 
focus on German Studies.

https://journals.uc.edu/index.php/fogs

durch den Brief einen »homosozialen« Raum, in dem der Leser und Wolf sich als Gleiche begegnen. $(137)$

Darüber hinaus böte sich unter den in diesem Beitrag aufgestellten Thesen und insbesondere nach erzähltheoretischen Analyseansätzen an, anerkannte (schriftliche) Quellen zum historischen Fall Friedrich Schwans sowie Abels Version einer (Re-)Konstruktion vergleichend hinzuzuziehen. Gerade das Mehrdeutige unterschiedlicher Erzählweisen ließe sich in einer solchen umfassenderen Untersuchung noch einmal differenzierter herausstellen. 
focus on German Studies.

https://journals.uc.edu/index.php/fogs

(ISSN 1076-5697)

\section{Literaturverzeichnis}

Abel, Jacob Friedrich. „Lebens-Geschichte Friedrich Schwans“. Sammlung und Erklärung merkwürdiger Erscheinungen aus dem menschlichen Leben. Zweiter Theil, Stuttgart, In der Erhardischen Buchhandlung, 1787, S. $1-86$.

Aurnhammer, Achim. „Engagiertes Erzählen. ,Der Verbrecher aus verlorener Ehre“،. Schiller und die böfische Welt, hrsg. von Achim Aurnhammer, Tübingen, Niemeyer, 1990, S. 254-270.

Avgerinou, Sophia. „Aufklärerische Botschaft und Erzähltechnik in Schillers Der Verbrecher aus verlorener Ehre“. German Life and Letters, vol. 68, 2015, S. 1-19.

Bangert, Sara, und Klaus Müller-Richter. „»Nur Taten sind ihnen untertan«. Subjektkonstitution durch Geständnis und Bekenntnis in Schillers Verbrecher aus verlorener Ehre“. Das Geständnis und seine Instanzen. Zur Bedeutungsverschiebung des Geständnisses im Prozess der Moderne, hrsg. von Anders Engberg-Pedersen, Wien, Turia + Kant, 2011, S. 271-292.

Bauer, Thomas. Die Vereindeutigung der Welt. Über den Verlust an Mehrdentigkeit und Vielfalt. 12th ed., Ditzingen, Philipp Reclam jun., 2018.

Biesdorf, Markus. Geheimnis und Aufklärung. Die Darstellung von Verbrechen in deutschsprachigen Texten 1782-1855. Tübingen, Narr Francke Attempto, 2016.

Dehrmann, Mark-Georg. „Literarische Tribunale. Der »Sonnenwirt« bei Schiller, Heinrich Ehregott Linck und Hermann Kurz“. Kriminalfallgeschichten, hrsg. von Alexander Košenina, München, Edition Text + Kritik, 2014, S. 130-150.

Deleuze, Gilles. Unterhandlungen. 1972-1990. 7. Ed., Frankfurt am Main, Suhrkamp, 2020.

Foucault, Michel. Überwachen und Strafen. Die Geburt des Gefängnisses. 21. Ed., Frankfurt am Main, Suhrkamp, 2019. 
focus on German Studies.

https://journals.uc.edu/index.php/fogs

focus

(ISSN 1076-5697)

on German Studies

Fulda, Daniel, und Stefan Matuschek. „Literarische Formen in anderen Diskursformationen: Philosophie und Geschichtsschreibung." Grenzen der Literatur. Zu Begriff und Phänomen des Literarischen, hrsg. von Jannidis Fotis, Gerhard Lauer und Simone Winko, Berlin \& New York, 2009, S. 188-219.

Geisenhanslüke, Achim. Literatur und Ehrlosigkeit. Paderborn, Wilhelm Fink, 2014.

High, Jeffrey L. „(A fragment of) A True Story (from most recent history): The Truth in Schiller's Literary Prose Works“. Schiller's Literary Prose Work. New Translations and Critical Essays, Rochester \& New York, Camden House, 2008, S. 173-187.

Höcker, Arne. „Der Fall der Literatur: Beobachten - Zitieren - Urteilen“. Paradigmatische Fälle. Konstruktion, Narration und Verallgemeinerung von Fall-Wissen in den Geistes- und Sozialwissenschaften, hrsg. von Ruben Hackler und Katherina Kinzel, Basel, Schwabe, 2016, S. 67-86.

---. „In Citation: „A Violation of the Law of Boundaries“ in Schiller and Kleist“. The Germanic Review, vol. 89, 2014, S. 60-75.

Kaul, Susanne. „Wie der Leser mit dem Held warm wird. Zu Schillers Verbrecher aus verlorener Ehre“. Sympathie und Literatur. Zur Relevanz, des Sympathiekonzeptes für die Literaturwissenschaft, hrsg. von Claudia Hillebrandt und Elisabeth Kampmann, Berlin, Erich Schmidt, 2014, S. 236-250.

Lehmann, Johannes F. „Erfinden, was der Fall ist. Fallgeschichte und Rahmen bei Schiller, Büchner und Musil“. Zeitschrift für Germanistik, vol. 19, 2009, S. 361-380.

---. Im Abgrund der Wut. Zur Kultur- und Literaturgeschichte des Zorns. Freiburg i.Br./Berlin/Wien, Rombach, 2012.

Lüdemann, Susanne. „Literarische Fallgeschichten. Schillers »Verbrecher aus verlorener Ehre« und Kleists »Michael Kohlhaas«". Das Beispiel. Epistemologie des Exemplarischen, hrsg. von Jens Ruchatz, Berlin, Kadmos, 2007, S. 208-223.

Martus, Steffen. „Verbrechen lohnt sich. Die Ökonomie der Literatur in Schillers Der Verbrecher aus Infamie“. Euphorion, vol. 99, 2005, S. 243-271. 
focus on German Studies.

https://journals.uc.edu/index.php/fogs

focus

(ISSN 1076-5697)

on German Studies

Nilges, Yvonne. „Homo Homini Lupus? A man in Wolf's clothing-Schiller's variations on a legal theme“. Oxford German Studies, vol. 38, 2009, S. 13-27.

Nünning, Ansgar. „,Verbal Fictions?‘: Kritische Überlegungen und narratologische Alternativen zu Hayden Whites Einebnung des Gegensatzes zwischen Historiographie und Literatur." Literaturwissenschaftliches Jahrbuch im Auftrage der Görres-Gesellschaft, vol. 40, Duncker \& Humblot, 1999, S. 351-380.

Ostermann, Eberhard. „Christian Wolfs Kampf um Anerkennung. Eine anerkennungstheoretische Deutung von Schillers Erzählung Der Verbrecher aus verlorener Ehre“. Literatur in Wissenschaft und Unterricht, vol. 34, 2001, S. 211-224.

Pethes, Nicolas. Literarische Fallgeschichten. Zur Poetik einer epistemischen Schreibweise. Konstanz, University Press, 2016.

Pfeiffer, Joachim. „Die Freiheit des republikanischen Subjekts. Zu Schillers Erzählung Der Verbrecher aus verlorener Ehre“. „W Whrheit, Sittlichkeit und Freiheit“. Schillers Aktualität in Schule und Hochschule, hrsg. von Holger Rudloff, Herbolzheim, Centaurus, 2006, S. 97-111.

Rybska, Agnieszka Magdalena. Deutsche Kriminalgeschichten von 1780 bis 1820 als Anfänge der Kriminalliteratur. Frankfurt am Main, Lang, 2011.

Schiller, Friedrich: „Der Verbrecher aus verlorener Ehre. Eine wahre Geschichte“. Friedrich Schiller. Sämtliche Werke, hrsg. von Gerhard Fricke und Herbert G. Göpfert, München, Hanser, 1962, S. 13-36.

Von Hoorn, Tanja. „Affektregie. Schillers Verbrecher aus Leidenschaft“. Die Erzähblung der Aufklärung. Beiträge zur DGEJ-Jahrestagung 2015 in Halle a. d. Saale, hrsg. von Berndt Frauke und Daniel Fulda, Hamburg, Felix Meiner, 2018, S. 554-563.

Weber, Philipp. „Abgrund zwischen den Zeilen. Die Stimme des Gewissens in der Prosa Kants und Schillers.“ Deutsche Vierteljahrsschrift für Literaturwissenschaft und Geistesgeschichte, vol. 94, 2020, S. 287-317.

White, Hayden. Tropics of Discourse. Essays in Cultural Criticism. 3. Ed., Baltimore \& Maryland, 1987. 